\title{
COVID-19 in CKD Patients: Lessons from 553 CKD Patients with Biopsy-Proven Kidney Disease
}

\author{
Juan León Román ${ }^{a} \quad$ Clara García-Carro $^{a} \quad$ Irene Agraz $^{a} \quad$ Nestor Toapanta ${ }^{a}$ \\ Ander Vergara $^{a, b} \quad$ Alejandra Gabaldón ${ }^{c}$ Irina Torres ${ }^{a}$ Roxana Bury ${ }^{a}$ \\ Cinthia Baldallo ${ }^{a}$ Daniel Serón ${ }^{a, b}$, María José Soler ${ }^{a, b}$,

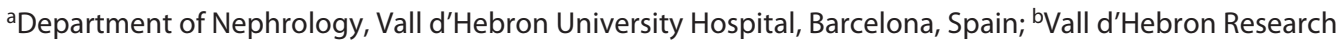 \\ Institute (VHIR), Nephrology Department, Nephrology Research Group, Hospital Universitari Vall d'Hebron, \\ Universitat Autònoma de Barcelona, Barcelona, Spain; 'Department of Pathology, Vall d'Hebron University Hospital, \\ Barcelona, Spain; 'Instituto Carlos IIIFEDER, Red de Investigación Renal (REDINREN), Madrid, Spain
}

\section{Keywords}

Coronavirus $\cdot$ COVID-19 - Severe acute respiratory syndrome coronavirus 2 . Chronic kidney disease . Biopsy-proven kidney disease

\footnotetext{
Abstract

Introduction: Chronic kidney disease (CKD) patients infected with COVID-19 are at risk of serious complications such as hospitalization and death. The prognosis and lethality of $\mathrm{CO}$ VID-19 infection in patients with established kidney disease has not been widely studied. Methods: Data included patients who underwent kidney biopsy at the Vall d'Hebron Hospital between January 2013 and February 2020 with COVID-19 diagnosis during the period from March 1 to May 15, 2020. Results: Thirty-nine (7\%) patients were diagnosed with COVID-19 infection. Mean age was $63 \pm 15$ years and $48.7 \%$ were male. Hypertension was present in $79.5 \%$, CKD without renal replacement therapy in $76.9 \%$, and cardiovascular disease in $64.1 \%$. Nasopharyngeal swab was performed
}

in 26 patients; older $(p=0.01)$, hypertensive $(p=0.005)$, and immunosuppressed $(p=0.01)$ patients, those using RASblocking drugs $(p=0.04)$, and those with gastrointestinal symptoms ( $p=0.02$ ) were more likely to be tested for COVID-19. Twenty-two patients required hospitalization and $15.4 \%$ died. In bivariate analysis, mortality was associated with older age $(p=0.03)$, cardiovascular disease $(p=0.05)$, chronic obstructive pulmonary disease $(p=0.05)$, and low hemoglobin levels $(p=0.006)$. Adjusted Cox regression showed that low hemoglobin levels at admission had 1.81 greater risk of mortality. Conclusions: Patients with COVID-19 infection and kidney disease confirmed by kidney biopsy presented a mortality of $15.4 \%$. Swab test for COVID-19 was more likely to be performed in older, hypertensive, and immunosuppressed patients, those using RAS-blocking drugs, and those with gastrointestinal symptoms. Low hemoglobin is a risk factor for mortality.

(C) 2021 The Author(s)

Published by S. Karger AG, Basel karger@karger.com www.karger.com $/ \mathrm{kbr}$

Karger $\stackrel{V}{\%}$

BOPEN ACCESS
C) 2021 The Author(s)

Published by S. Karger AG, Basel

This is an Open Access article licensed under the Creative Common Attribution-NonCommercial-4.0 International License (CC BY-NC) (http://www.karger.com/Services/OpenAccessLicense), applicable to the online version of the article only. Usage and distribution for commercial purposes requires written permission.
Correspondence to:

María José Soler, mjsoler01@gmail.com 


\section{Introduction}

Severe acute respiratory syndrome coronavirus 2 (SARS-CoV-2) is a beta-coronavirus responsible for respiratory infection with variable clinical expression, from its most frequent asymptomatic presentation to severe pneumonia complicated by acute respiratory distress syndrome (ARDS), multiple organ failure, and death [1, 2]. Transmission occurs through respiratory drops from person to person; even presymptomatic people are infectious [1]. The virus enters into human cells through the angiotensin-converting enzyme receptor [1] and has a high tropism toward lung cells [2]. The first cases were described in Wuhan, China, and it was classified as an international health emergency in March 2020 by the WHO due to its high rate of infection and inherent complications [2]. Research has focused on preventing its spread and trying to establish pharmacological treatments. There are studies with various drugs tested; until now, ventilatory support has been the fundamental basis of treatment in severe cases that develop adult respiratory distress syndrome [2].

Several studies have focused on investigating the risk factors associated with developing ARDS [3]. Factors related to increased risk of complications and death are age over 65 years, cardiovascular disease, lung disease, hypertension, diabetes, and liver disease [3], as well as analytical alterations such as neutrophilia, elevation of acute phase reactants, and organic and coagulation dysfunction [4].

Chronic kidney disease (CKD) is described as a risk factor for both increased susceptibility to infection and the development of serious complications [5]. The impact of COVID-19 infection at renal level is poorly understood, and some authors have described acute kidney injury associated with COVID-19 infection evidencing involvement in glomerular cells with quantification of viral genetic material in histological studies of deceased patients diagnosed with COVID-19 [6]. Among patients with renal replacement therapy, the Goicoechea group [7] described a high mortality rate with 11 (30.5\%) of 36 patients with COVID-19 infection and ESRD on hemodialysis. In addition, mortality was associated with analytical alterations such as elevation of lactate dehydrogenase and C-reactive protein. Studying COVID-19, the Registry of the Spanish Society of Nephrology, SánchezÁlvarez et al. [8] demonstrated higher mortality in hemodialysis patients compared to those with kidney transplantation after COVID-19 infection. Interestingly, kidney transplant patients with COVID-19 infection presented rapid clinical progression associated with de- crease in CD3, CD4, and CD8 cells by immunosuppressive drugs for preventing graft rejection [9].

There is limited scientific literature regarding COVID-19 infection and CKD, specifically in patients with glomerular and tubular kidney disease. The objective of this study is to determine general characteristics, analytical studies, and clinical evolution of patients with CKD in whom a percutaneous renal biopsy has been performed at the Vall d'Hebron Hospital and who have presented COVID-19 infection and to identify risk factors for hospitalization and mortality of those patients.

\section{Materials and Methods}

Medical records of 553 patients who underwent kidney biopsy at Vall d'Hebron Hospital between January 2013 and December 2019 were reviewed. In all biopsied patients, COVID-19 infection was assessed by reviewing clinical records and nasopharyngeal swabs for SARS-COV-2 from March 2020 to May 15, 2020. Clinical and analytical data were collected from patients who had high clinical suspicion of COVID-19 infection as well as clinical course after infection (see online suppl. Fig. 1; for all online suppl. material, see www.karger.com/doi/10.1159/000515714). High clinical suspicion of COVID-19 infection in absence of a nasal swab was defined as the clinical diagnosis of those patients evaluated by a primary care physician who presented fever, gastrointestinal symptoms, and respiratory symptoms associated with contact with a patient diagnosed of COVID-19 infection. The Ethics Committee of Vall d'Hebron University Hospital approved the study protocol (PR [AG]5713/2020).

Demographics, clinical variables, and analytical results were obtained from the hospital computer system. The quantitative variables age, BMI, number of lymphocytes, platelets, and hemoglobin presented a normal distribution. The normality of the variables was assessed by the Kolmogorov-Smirnov test.

Qualitative variables are presented with their frequency distribution. Quantitative variables are presented with their mean with standard deviation or median with interquartile range, 25-75\%. First, we determined the distribution of the variables with the Kolmogorov-Smirnov test. The qualitative variables were analyzed with the $\chi^{2}$ test or Fisher's exact coefficient. The relationship between 2 quantitative variables was analyzed with the Spearman test and quantitative variables with the Student's $t$ test. Bivariate and multivariate analyses were obtained with logistic regression. The Cox regression analysis was used to identify mortality risk factors in patients with high suspicion of COVID-19 infection. Statistical studies were processed with the SPSS version 20 program.

\section{Results}

Thirty-nine of the 553 patients have been diagnosed with COVID-19 infection (either by swab confirmation or high clinical suspicion) since March 2020. The average 
Table 1. Demographic and clinical characteristics of the 39 patients

\begin{tabular}{|c|c|c|c|c|}
\hline Variable & Total & $\begin{array}{l}\text { Survivors } \\
(n=33)\end{array}$ & $\begin{array}{l}\text { Nonsurvivors } \\
(n=6)\end{array}$ & $p$ value \\
\hline Age, years & $63 \pm 15$ & $61 \pm 14.9$ & $75 \pm 9.3$ & 0.03 \\
\hline Male & $19(48.7)$ & $15(45.5)$ & $4(66.7)$ & 0.34 \\
\hline \multicolumn{5}{|l|}{ Ethnicity } \\
\hline Caucasian & $32(82.1)$ & $26(78.8)$ & $6(100)$ & \\
\hline Latin & $6(15.4)$ & $6(18.2)$ & $0(0)$ & 0.46 \\
\hline Asian & $1(2.6)$ & $1(3.0)$ & $0(0)$ & \\
\hline $\mathrm{BMI}, \mathrm{kg} / \mathrm{m}^{2 *}$ & $28.8 \pm 6.5$ & $29.2 \pm 6.8$ & $25.6 \pm 4.0$ & 0.23 \\
\hline Tobacco & $13(33.3)$ & $10(30.3)$ & $3(50.0)$ & 0.35 \\
\hline Hypertension & $31(79.5)$ & $25(75.8)$ & $6(100)$ & 0.18 \\
\hline Diabetes & $19(48.7)$ & $17(51.5)$ & $2(33.3)$ & 0.41 \\
\hline Cardiovascular disease & $25(64.1)$ & $19(57.6)$ & $6(100)$ & 0.05 \\
\hline Chronic obstructive pulmonary disease & $8(20.5)$ & $5(15.2)$ & $3(50.0)$ & 0.05 \\
\hline Rheumatologic disease & $8(20.5)$ & $6(18.2)$ & $2(33.3)$ & 0.44 \\
\hline Use of RAS-blocking drugs & $18(46.2)$ & $18(54.5)$ & $0(0)$ & 0.01 \\
\hline CKD not on RRT & $30(76.9)$ & $25(75.8)$ & $5(83.3)$ & 0.69 \\
\hline Kidney replace therapy & $9(23.1)$ & $8(20.5)$ & $1(2.6)$ & 0.69 \\
\hline Dialysis & $4(10.3)$ & $3(9.1)$ & $1(16.7)$ & 0.57 \\
\hline Kidney transplant & $5(12.8)$ & $5(12.8)$ & $0(0)$ & 0.31 \\
\hline Immunosuppression & $17(43.6)$ & & & \\
\hline Prednisone & $15(38.5)$ & & & \\
\hline Mycophenolate & $7(17.9)$ & & & \\
\hline Tacrolimus & $6(15.4)$ & $13(39.4)$ & $4(66.7)$ & 0.22 \\
\hline Rituximab & $1(2.6)$ & & & \\
\hline Omalizumab & $1(2.6)$ & & & \\
\hline Everolimus & $1(2.6)$ & & & \\
\hline SARS-CoV-2 swab test & $26(66.7)$ & $20(60.6)$ & $6(100)$ & 0.06 \\
\hline \multicolumn{5}{|l|}{ Symptoms } \\
\hline Fever & $26(66.7)$ & $22(66.7)$ & $4(66.7)$ & 1.00 \\
\hline Myalgia & $18(46.2)$ & $17(51.5)$ & $1(16.7)$ & 0.12 \\
\hline Diarrhea, nausea/vomiting & $9(23.1)$ & $7(21.2)$ & $2(33.3)$ & 0.52 \\
\hline Image-proven pneumonia & $19(48.7)$ & $33(100)$ & $6(100)$ & 0.34 \\
\hline Hospitalization & $22(56.4)$ & $17(51.5)$ & $5(83.3)$ & 0.15 \\
\hline \multicolumn{5}{|l|}{ Treatment } \\
\hline Hydroxychloroquine & $20(51.3)$ & $17(51.5)$ & $3(50.0)$ & 0.95 \\
\hline Lopinavir/ritonavir & $15(38.5)$ & $12(36.4)$ & $3(50.0)$ & 0.53 \\
\hline Azithromycin & $23(59.0)$ & $19(57.6)$ & $4(66.7)$ & 0.68 \\
\hline Tocilizumab & $6(15.4)$ & $4(12.1)$ & $2(33.3)$ & 0.19 \\
\hline High-dose corticosteroids & $9(23.1)$ & $6(18.2)$ & $3(50.0)$ & 0.09 \\
\hline
\end{tabular}

Variables with normal distribution are expressed as mean \pm standard deviation. Qualitative variables are expressed as $n(\%)$. Bold indicates significant $p$ values of $\leq 0.05$. ${ }^{*}$ BMI in 32 of 39 patients. BMI, body mass index; RAS, renin-angiotensin system; CKD, chronic kidney disease; RRT, renal replacement therapy.

age was $63 \pm 15$ years and $48.7 \%$ were male. Demographic and clinical characteristics are shown in Table 1 in the total studied subjects, survivors, and nonsurvivors. In brief, hypertension was present in $79.5 \%$ of patients, CKD without renal replacement therapy in $76.9 \%$, and cardiovascular disease in $64.1 \%$. The most frequent symptoms were fever (66.7\%) and myalgias (46.2\%). Twenty-two percent of patients required hospital admission, of which
51.3\% received hydroxychloroquine, 38.5\% lopinavir/ ritonavir, 59.0\% azithromycin, $15.4 \%$ tocilizumab, and $23.1 \%$ high-dose corticosteroids. In our studied patients, $11.4 \%(4 / 31)$ of lupus nephritis, $3.3 \%$ (2/59) of IgAN, and none of $(0 / 31)$ membranous patients were diagnosed of COVID-19 infection. Membranous nephropathy patients seem to be protected against COVID-19 infection; however, statistical differences among groups were not 


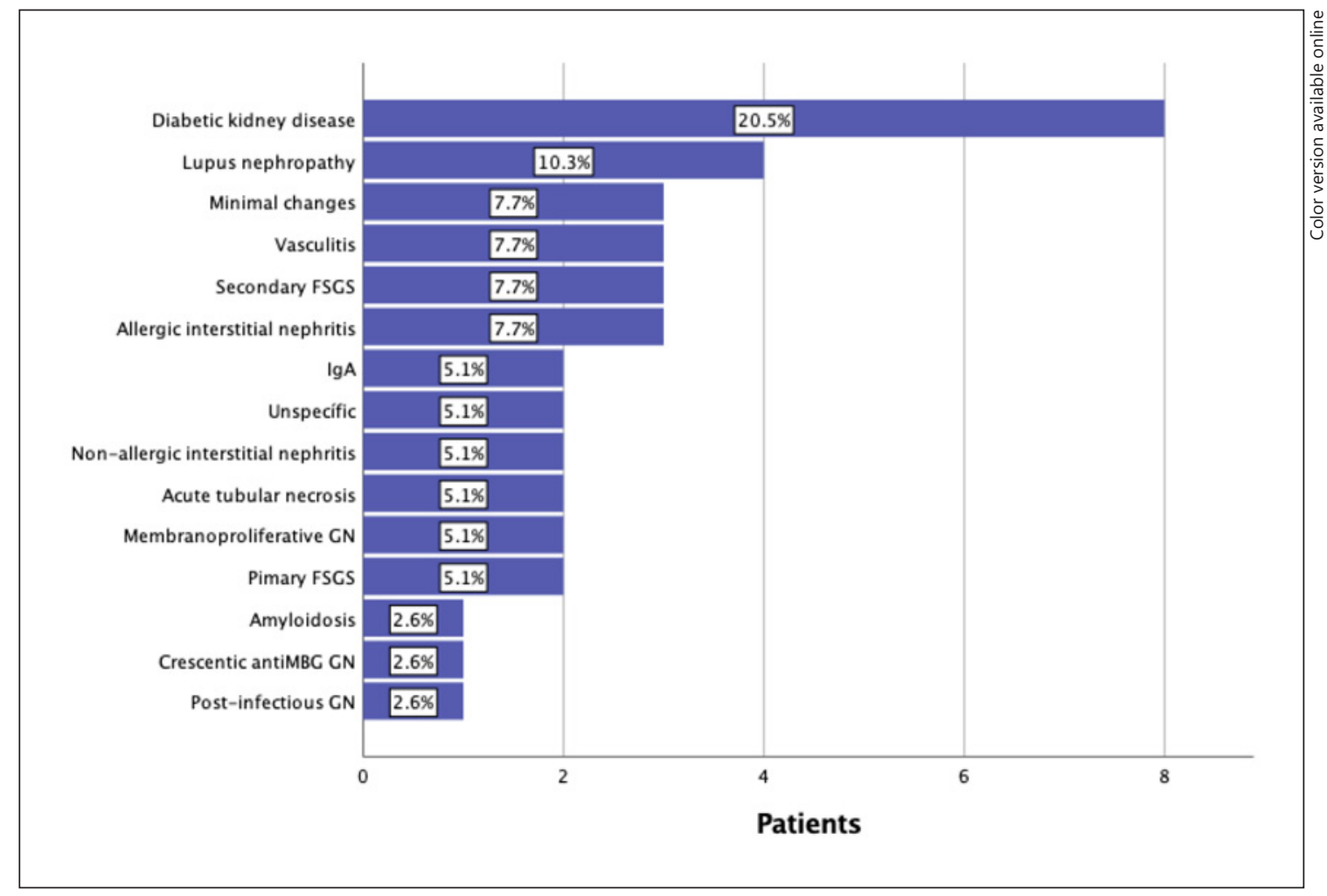

Fig. 1. Histological diagnoses of the renal biopsies of the 39 patients. IgA, IgA nephropathy; GN, glomerulonephritis; FSGS, Focal Segmental Glomerulosclerosis.

detected $(p=0.07)$. Of all studied subjects, 15 patients (38.5\%) with CKD without KRT and 7 (17.9\%) patients with KRT were admitted to the hospital. There were no significant differences regarding the risk of hospitalization and death between CKD patients with and without $\operatorname{KRT}(p=0.14$ and $p=0.69$, respectively). In addition, there was no significant difference in mortality between CKD stages $1-3$ and CKD stages $4-5$ ( $p=0.53$ ). Histological diagnosis of kidney biopsies was as follows: diabetic kidney disease (20.5\%), lupus nephropathy (10.3\%), minimal change disease, allergic interstitial nephritis, and secondary FSGS (7.7\%), and vasculitis (7.7\%) (see Fig. 1). Of note, 17 patients received immunosuppression at $\mathrm{CO}$ VID-19 diagnosis, 12 for their underlying glomerular disease and 5 of them to prevent allograft kidney rejection. There were no significant differences in mortality between patients with and without immunosuppression. Swab was performed in 26 patients $(66.7 \%)$ with COVID-19 suspicion. In the bivariate analysis, older age ( $p=$ $0.01)$, hypertensive $(p=0.005)$, and immunosuppressed $(p=0.01)$ patients, those using RAS-blocking drugs $(p=$ 0.04 ), and those with gastrointestinal symptoms ( $p=$ 0.02 ) were more likely be tested for COVID-19 than those with the rest of comorbidities and clinical manifestations (Table 2).

Analytical results of patients with hospital admission are depicted in Table 3 . The mortality rate was $15.4 \%$. In the actuarial survival analysis, chronic obstructive pulmonary disease (COPD) was significantly increased in nonsurvivors as compared with survivors, and use of RAS blockade drugs was significantly increased in survivors as compared with nonsurvivors (online suppl. Fig. 2-5). The Cox regression analysis suggested that nonsurviving COVID-19 patients had a lower hemoglobin levels at admission as compared to surviving COVID-19 patients (Table 4). Low hemoglobin did not statistically correlate with other laboratory parameters such as D-dimer, C-reactive protein, and IL- $6(p=0.23, p=-0.27$, and $p=0.33$, respectively).

\section{Discussion/Conclusions}

Our study evaluates the prevalence, clinical characteristics, and prognosis of patients followed after kidney biopsy in our outpatient consult with high suspicion or in- 
Table 2. Clinical characteristics of patients with and without SARS-CoV-2 swab test

\begin{tabular}{lccc}
\hline Variable & $\begin{array}{l}\text { Without } \\
\text { SARS-CoV-2 } \\
\text { swab }(n=13)\end{array}$ & $\begin{array}{l}\text { SARS-CoV-2 } \\
\text { swab positive } \\
(n=26)\end{array}$ & $p$ value \\
\hline Age, years & $55 \pm 17$ & $68 \pm 12$ & $\mathbf{0 . 0 1}$ \\
Male & $6(46.2)$ & $13(50.0)$ & 0.82 \\
BMI, kg/m ${ }^{2 *}$ & $27.1 \pm 7.1$ & $29.1 \pm 6.34$ & 0.41 \\
Tobacco & $3(23.1)$ & $10(38.5)$ & 0.34 \\
Hypertension & $7(53.8)$ & $24(92.3)$ & $\mathbf{0 . 0 0 5}$ \\
Diabetes & $4(30.8)$ & $15(57.7)$ & 0.11 \\
Cardiovascular disease & $6(46.2)$ & $19(73.1)$ & 0.09 \\
Chronic obstructive pulmonary disease & $2(15.4)$ & $6(23.1)$ & 0.58 \\
Rheumatologic disease & $0(0)$ & $8(30.1)$ & 0.22 \\
Use of RAS-blocking drugs & $9(69.2)$ & $9(34.6)$ & $\mathbf{0 . 0 4}$ \\
CKD without RRT & $12(92.3)$ & $18(69.2)$ & 0.11 \\
Dialysis & $0(0)$ & $4(15.4)$ & 0.14 \\
Kidney transplant & $1(7.7)$ & $5(19.2)$ & 0.35 \\
Dead & $0(0)$ & $6(23.1)$ & 0.06 \\
Immunosuppression & $2(15.4)$ & $15(57.7)$ & $\mathbf{0 . 0 1}$ \\
Symptoms & & & \\
$\quad$ Fever & $7(53.8)$ & $19(73.1)$ & 0.23 \\
Myalgia & $6(46.2)$ & $12(46.2)$ & 1.00 \\
Diarrhea, nausea/vomiting & $0(0)$ & $9(34.6)$ & $\mathbf{0 . 0 2}$ \\
\hline
\end{tabular}

* BMI in 32 of 39 patients. BMI, body mass index. Bold indicates significant $p$ values of $\leq 0.05$.

Table 3. Comparison of analytical results of the 22 patients hospitalized for COVID-19

\begin{tabular}{lccc}
\hline Variable & Total & Survivors & Nonsurvivors \\
\hline Leukocytes $\times 10^{9} / \mathrm{L}$ & $7.84(4.46-9.23)$ & $7.40(3.85-9.55)$ & $8.43(6.35-10.36)$ \\
Neutrophils $\times 10^{9} / \mathrm{L}$ & $5.80(2.70-7.90)$ & $5.40(2.25-8.39)$ & $6.20(4.10-9.50)$ \\
Lymphocytes $\times 10^{9} / \mathrm{L}$ & $0.89 \pm 0.55$ & $0.82 \pm 0.49$ & $1.16 \pm 0.72$ \\
Platelets $\times 10^{9} / \mathrm{L}$ & $175.36 \pm 61.0$ & $176.47 \pm 62.84$ & $171.60 \pm 61.03$ \\
Creatinine, $\mathrm{mg} / \mathrm{dL}$ & $1.99(1.32-3.78)$ & $2.04(1.25-3.68)$ & $1.73(1.23-4.48)$ \\
Hemoglobin, g/dL & $11.76 \pm 1.60$ & $12.25 \pm 1.17$ & $10.12 \pm 1.89$ \\
D-dimer, ng/mL & $395.50(157.50-623.50)$ & $406.0(181.0-619.0)$ & $1,024.33(169.99-1,521.50)$ \\
C-reactive protein, mg/dL & $5.75(2.86-15.58)$ & $5.07(2.72-15.48)$ & 0.71 \\
Interleukin, $6 \mathrm{pg} / \mathrm{mL}$ & $38.60(22.62-85.34)$ & $37.45(21.06-69.88)$ & $1,475.16(68.32-2882.0)$ \\
Ferritin, ng/mL & $718(561.50-1,292.75)$ & $718.0(576.50-1,193.75)$ & $926.50(506.0-1,347.0)$ \\
Lactate dehydrogenase, UI/L & $318.50(220.25-443.0)$ & $304.0(224.50-411.50)$ & $370.50(266.0-510.50)$ \\
\hline
\end{tabular}

Leukocytes $n=22$ (17 survivors and 5 nonsurvivors), platelets $n=22$ (17 survivors and 5 nonsurvivors), hemoglobin $n=22$ ( 17 survivors and 5 nonsurvivors), D-dimer $n=20$ (17 survivors and 3 nonsurvivors), CRP $n=19$ (16 survivors and 3 nonsurvivors), IL-6 $n=$ 17 ( 15 survivors and 2 nonsurvivors), ferritin $n=16$ ( 14 survivors and 2 nonsurvivors), and LDH $n=20$ ( 16 survivors and 4 nonsurvivors). Bold indicates significant $p$ values of $\leq 0.05$. Variables with normal distribution are expressed as mean \pm standard deviation. Variables with abnormal distribution are presented in median (IQ25\%-IQ75\%).

fection confirmed by COVID-19. Data from the literature in this population are scarce. In our cohort, the prevalence of COVID-19 was $7.05 \%$ (39 out of 553 patients), and the mortality rate was $15.4 \%$. In comparison with other series, our mortality rate was low $[7,8]$, and this may be ascribed in part to the fact that the majority of patients were kidney disease patients without ESRD. Recent publications from Williamson et al. [10] indicate that dialysis or ESRD highly increases mortality (HR 3.69) of COVID-19-infected patients. In concordance with 
Table 4. Adjustment of variables and Cox regression of characteristics with significant differences associated with mortality

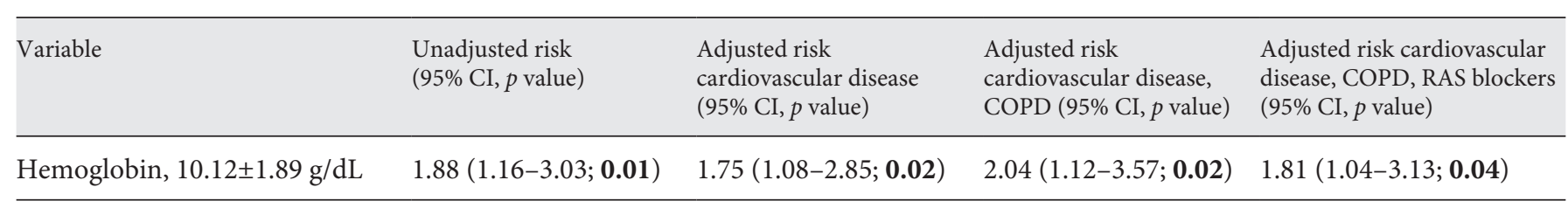

$\mathrm{CI}$, confidence interval; COPD, chronic obstructive pulmonary disease; RAS, renin-angiotensin system. Bold indicates significant $p$ values of $\leq 0.05$.

Goicoehea et al. [7] and Sánchez et al. [8], there were no significant differences in mortality between gender. $\mathrm{Wu}$ et al. [4] described in their cohort study of 201 patients that those who developed ARDS secondary to COVID-19 infection were older patients and had hypertension and diabetes as comorbidities. In our bivariate analysis, age $>65$ years, cardiovascular disease, and chronic obstructive pulmonary disease were identified as risk factors for mortality in our COVID-19 patients.

Interestingly, nasopharyngeal PCR of COVID-19 was only performed in 26 patients $(66.7 \%)$, which may be related to the overwhelming and containment period of the pandemic disease. Among them, older age, male sex, COPD, hypertension, diabetes, and hypercholesterolemia have been identified as risk factors of worse clinical prognosis and the need of intensive care unit admission as Grasselli et al. [11] defined in their cohort study of 3,988 patients admitted in the intensive care unit. Our results suggest that patients at risk for severe disease are admitted and subsequently tested in hospitals [12]. As expected, none of our COVID-19 infection suspicion patients without swab diagnosis died, reinforcing again the idea that the nonswab patients were patients with low risk of severe disease.

The beneficial/deleterious effects of RAS blockade have been under discussion since the COVID-19 outbreak [13-15]. However, Fan et al. [16] supported the deleterious effect of RAS blockade therapy in the COVID pandemic era in terms of increased infection and severity of the disease; Sparks et al. [17] in agreement with several scientific societies recommended to continue RAS blockade in front of the absence of evidence to support its discontinuation $[16,17]$. After that, several studies come out analysing the RAS effect on COVID-19 patients or patients at risk for COVID-19. Liu et al. [14] showed a possible RAS blockade benefit in terms of protecting against acute distress syndrome in patients older than 65 years and hypertension. In concordance, Reynolds et al. [15] demonstrated that previous treatment with medications acting on the RAAS was not associated with a higher risk of testing positive for COVID-19 or higher risk of severe COVID-19. Our study also suggests in part the nondeleterious effect of RAS blockade in renal patients in the new COVID-19 pandemic era.

Fever was presented in $66.7 \%$, myalgia in $46.2 \%$, gastrointestinal symptoms in $23.1 \%$, and $48.7 \%$ presented pneumonia evidenced by radiography and/or chest tomography. In addition, according to the WHO classification, $48.7 \%$ of patients presented mild clinical symptoms and $17.9 \%$, critical disease with septic shock. Our findings were similar to those of other authors regarding the high prevalence of fever and myalgias $[7,8]$. One of the limitations of this study is that we had only collected data on fever, myalgia, and gastrointestinal symptoms as clinical expressions of COVID-19, so we cannot compare other symptoms and signs such as oxygen desaturation, cough, and time of evolution of the clinical condition. Wu et al. [4] also described analytical risk factors to the development of ARDS, and progression from ARDS to death included neutrophilia and organ and coagulation dysfunction (higher LDH and D-dimer); by contrast, our study only found low hemoglobin levels as a risk factor for increased mortality. Interestingly, this result has not been widely observed in other series [18].

There is no consensus on the optimal treatment in patients with COVID-19 infection; likewise, the treatment in patients with glomerular and tubular diseases is established under general protocols and adjusted renal function of each patient. Treatments administered in our patients were based on the protocol designed by the Infectious Diseases team at the Vall d'Hebron Hospital. In the first pandemic wave, COVID-19 treatment in case of pneumonia without severity criteria included lopinavir/ ritonavir, hydroxychloroquine, and azithromycin; tocilizumab was reserved for those patients with pneumonia, adult respiratory distress syndrome, interleukin-6 levels $>40 \mathrm{pg} / \mathrm{mL}$, and/or D-dimer $>1,500 \mathrm{ng} / \mathrm{mL}$. Treatment with high doses of intravenous corticosteroids was re- 
served for patients with severe respiratory failure and absence of response to tocilizumab.

Our study has some limitations. First, the retrospective character of the study does not allow us to establish causality. Second, interpretation of our results is limited by the small sample size. Third, risk factors between analytical results could not be accurately analyzed due to the absence of D-dimer, IL-6, ferritin, and LDH in all patient outcomes. Despite this, we were able to create a model that exposes risk or protective factors and mortality.

In conclusion, our study showed a decreased mortality compared to other series with more advanced CKD and COVID-19 infection where dialysis or ESRD highly increased the mortality rate. Swab test for COVID-19 was more likely to be performed in older, hypertensive, and immunosuppressed patients and those with gastrointestinal symptoms. In addition, it is important to mention that nonswab patients with COVID-19 disease presented a milder disease without any case of death. In our study, low hemoglobin levels at admission were identified as risk factors for mortality in COVID-19 patients with kidney disease.

\section{Acknowledgment}

The article is dedicated to all health care personnel who have contributed to the investigation and treatment of COVID-19 infection, as well as in honor of all patients who died fighting the pandemic.

\section{Statement of Ethics}

The study protocol was approved by the Ethics Committee of Vall d'Hebron University Hospital and was conducted in full accordance with the principles of the Declaration of Helsinki PR (AG) 5713/2020. Informed consent for renal biopsy was signed for each patient. Consent for this retrospective study was not needed.

\section{Conflict of Interest Statement}

M.J.S. reports conflicts of interest with fees from NovoNordisk, personal fees from Janssen, grants and nonfinancial support from Boehringer, nonfinancial support from Eli Lilly, personal fees from AstraZeneca, nonfinancial support from Esteve, personal fees from Fresenius, personal fees from Mundipharma, personal fees from Pfizer, personal fees from Bayer, and personal fees from Vifor, outside the submitted work. The results presented in this article have not been published previously in whole or part, except in abstract format.

\section{Funding Sources}

The authors are current recipients of research grants from the Fondo de Investigación Sanitaria-Feder (FI17/00257 and EIN20201123381) and Redinren (RD16/0009/0030).

\section{Author Contributions}

J.L., C.G., I.A., R.B., and C.B. analyzed the current literature, provided critical feedback, and helped to draft the manuscript. N.T., A.V., and I.T. reviewed the database and designed the tables and figures. A.G. provided kidney biopsies feedback. M.J.S. and D.S. conceived the idea, carried out the implementation, and supervised the drafting.

\section{References}

1 Gandhi RT, Lynch JB, del Rio C. Mild or moderate Covid-19. N Engl J Med. 2020;383(18): 1757-66.

2 World Health Organization. Clinical management of COVID-19;2020. Avaliable from: https: //www.who.int/publications/i/item/ clinical-management-of-covid-19 Accessed 2020 Jul.

3 Huang C, Wang Y, Li X, Ren L, Zhao J, Hu Y, et al. Clinical features of patients infected with 2019 novel coronavirus in Wuhan, China. Lancet. 2020;395(10223):497-506.

4 Wu C, Chen X, Cai Y, Xia J, Zhou X, Xu S, et al. Risk factors associated with acute respiratory distress syndrome and death in patients with coronavirus disease 2019 pneumonia in Wuhan, China. JAMA Intern Med. 2020; 180(7):934-43.
5 Henry BM, Lippi G. Chronic kidney disease is associated with severe coronavirus disease 2019 (COVID-19) infection. Int Urol Nephrol. 2020;52(6):1193-4.

6 Puelles VG, Lütgehetmann M, Lindenmeyer MT, Sperhake JP, Wong MN, Allweiss L, et al. Multiorgan and renal tropism of SARSCoV-2. N Engl J Med. 2020;383:590-2.

7 Goicoechea M, Sánchez Cámara LA, Macías N, Muñoz de Morales A, Rojas ÁG, Bascuñana A, et al. COVID-19: clinical course and outcomes of 36 hemodialysis patients in Spain. Kidney Int. 2020;98(1):27-34.

8 Sánchez-Álvarez JE, Fontán MP, Martín CJ, Blasco Pelícano M, Cabezas Reina CJ, Sevillano Prieto ÁM, et al. Status of SARS-CoV-2 infection in patients on renal replacement therapy. Report of the COVID-19 registry of the Spanish society of nephrology (SEN). Nefrología. 2020;40(3):272-8.
9 Akalin E, Azzi Y, Bartash R, Seethamraju H, Parides M, Hemmige V, et al. Covid-19 and kidney transplantation. N Engl J Med. 2020; 382:2475-7.

10 Williamson EJ, Walker A, Goldacre B. Factors associated with COVID-19-related death using opensafely. Nature. 2020;584:430-6.

11 Grasselli G, Greco M, Zanella A, Albano G, Antonelli M, Bellani G, et al. Risk Factors associated with mortality among patients with COVID-19 in intensive care units in Lombardy, Italy. JAMA Intern Med. 2020;180(10): 1345-55.

12 Chen T, Wu D, Chen H. Clinical characteristics of 113 deceased patients with coronavirus disease 2019: retrospective study. BMJ. 2020; 368:m1091. 
13 Wang R, Liao C, He H, Hu C, Wei Z, Hong Z, et al. COVID-19 in hemodialysis patients: a report of 5 cases. Am J Kidney Dis. 2020; 76(1):141-3.

14 Liu Y, Huang F, Xu J, Yang P, Qin Y, Cao M, et al. Antihypertensive angiotensin II receptor blockers associated to mitigation of disease severity in elderly COVID-19 patients. Medrxiv. 2020. Available from: https://www.medrxiv.org/content/10.1101/2020.03.20.20039 586v1.
15 Reynolds HR, Adhikari S, Pulgarin C, Troxel $\mathrm{AB}$, Iturrate $\mathrm{E}$, Johnson $\mathrm{SB}$, et al. Renin-angiotensin-aldosterone system inhibitors and risk of Covid-19. N Engl J Med. 2020;382: 2441-8.

16 Fang L, Karakiulakis G, Roth M. Are patients with hypertension and diabetes mellitus at increased risk for COVID-19 infection? Lancet Respir Med. 2020;8(4):e21.
17 Sparks MA, South A, Welling P, Luther JM, Cohen J, Byrd JB, et al. Sound science before quick judgement regarding RAS blockade in COVID-19. Clin J Am Soc Nephrol. 2020; 15(5):714-6.

18 Banerjee A, Pasea L, Harris S, Gonzalez-Izquierdo A, Torralbo A, Shallcross L, et al. Estimating excess 1 -year mortality associated with the COVID-19 pandemic according to underlying conditions and age: a populationbased cohort study. Lancet. 2020;395(10238): 1715-25. 